\title{
Homotopy analysis method for fuzzy Boussinesq equation
}

\author{
Amir Fallahzadeh ${ }^{1}$ Mohammad Ali Fariborzi Araghi ${ }^{1}$
}

Received: 27 October 2014 / Accepted: 15 June 2015/Published online: 8 July 2015

(C) The Author(s) 2015. This article is published with open access at Springerlink.com

\begin{abstract}
In this work, the fuzzy Boussinesq equation is considered to solve via the homotopy analysis method (HAM). For this purpose, a theorem is proved to illustrate the convergence of the proposed method. Also, two sample examples are solved by applying the HAM to verify the efficiency and importance of the method.
\end{abstract}

Keywords Homotopy analysis method · Fuzzy

Boussinesq equation · Fuzzy numbers · Convergence

\section{Introduction}

In recent years, some numerical and analytical methods were proposed in order to solve fuzzy differential equations $[1-8,10,18,19]$. One of the powerful semi-analytical methods to solve differential equations is the homotopy analysis method (HAM). In [14], the authors applied this method to solve the Boussinesq equation in crisp case. In this work, we consider the fuzzy form of Boussinesq equation as follows:

$$
\widetilde{u}_{t t}+\alpha \widetilde{u}_{x x}+\beta\left(\widetilde{u}^{2}\right)_{x x}-\widetilde{u}_{x x x x}=\widetilde{0}, \quad 0 \leq t \leq T, x>0 .
$$

Amir Fallahzadeh

amir_falah6@yahoo.com

Mohammad Ali Fariborzi Araghi

fariborzi.araghi@gmail.com; m_fariborzi@iauctb.ac.ir

1 Department of Mathematics, Islamic Azad University, Central Tehran Branch, P.O. Box 13185.768, Tehran, Iran
With the following initial conditions,

$\widetilde{u}(x, 0)=\widetilde{f}(x)$,

$\widetilde{u}_{t}(x, 0)=\widetilde{g}(x)$,

where $\widetilde{u}$ is unknown fuzzy function, $\alpha$ and $\beta$ are crisp constant coefficients and $\widetilde{f}$ and $\widetilde{g}$ are known fuzzy functions.

In order to solve Eq. (1), we apply the HAM in fuzzy case as an important and efficient method to find the solution of differential equations. The HAM, proposed by Liao, [16, 17], is a semi-analytical method which the solution is obtained as a series form according to a recursive relation stems from a deformation equation [13, 14].

In Sect. 2, we remind some fuzzy concepts briefly. In Sect. 3, we apply the HAM to solve the fuzzy Boussinesq equation and we prove a theorem to show the convergence of the proposed method. In Sect. 4, we solve two sample fuzzy Boussinesq equations and we obtain a series solution by this method.

\section{Preliminaries}

In this section, we recall some basic definitions of fuzzy sets theory [22].

Definition 2.1 A fuzzy parametric number $u$ is a pair $(\underline{u}(r), \bar{u}(r)), \quad 0 \leq r \leq 1$, which satisfies the following requirements:

1. $\underline{u}(r)$ is a bounded left continuous non-decreasing function over $[0,1]$,

2. $\bar{u}(r)$ is a bounded left continuous non-increasing function over $[0,1]$,

3. $\underline{u}(r) \leq \bar{u}(r), \quad 0 \leq r \leq 1$. 
The set of all these fuzzy numbers is denoted by $\mathbb{E}^{1}$. For $u=(\underline{u}, \bar{u}), v=(\underline{v}, \bar{v}) \in \mathbb{E}^{1}, \quad k \in \mathbb{R}$ the addition, multiplication, and the scaler multiplication of fuzzy numbers are defined by

$$
\begin{aligned}
& (\underline{u+v})(r)=\underline{u}(r)+\underline{v}(r), \\
& (\overline{u+v})(r)=\bar{u}(r)+\bar{v}(r), \\
& (\underline{u \cdot v})(r)=\min \{\underline{u}(r) \cdot \underline{v}(r), \underline{u}(r) \cdot \bar{v}(r), \bar{u}(r) \cdot \underline{v}(r), \bar{u}(r) \cdot \bar{v}(r)\}, \\
& (\overline{u \cdot v})(r)=\max \{\underline{u}(r) \cdot \underline{v}(r), \underline{u}(r) \cdot \bar{v}(r), \bar{u}(r) \cdot \underline{v}(r), \bar{u}(r) \cdot \bar{v}(r)\}, \\
& \underline{k u}(r)=k \underline{u}(r), \overline{k u}(r)=k \bar{u}(r), \quad k \geq 0, \\
& \underline{k u}(r)=k \bar{u}(r), \overline{k u}(r)=k \underline{u}(r), \quad k \leq 0 .
\end{aligned}
$$

Definition 2.2 A fuzzy parametric number $\widetilde{u}$ is positive (negative) if and only if $\underline{u}(r) \geq 0(\bar{u}(r) \leq 0) \quad \forall r \in[0,1]$.

Remark 2.3 If fuzzy parametric numbers $\widetilde{u}$ and $\widetilde{v}$ are positive, then $\widetilde{u} \cdot \widetilde{v}(r)=(\underline{u}(r) \underline{v}(r), \bar{u}(r) \bar{v}(r))$.

Definition 2.4 A function $f: \mathbb{R}^{1} \longrightarrow \mathbb{E}^{1}$ is called a fuzzy function. If for arbitrary fixed $t_{0} \in \mathbb{E}^{1}$ and $\varepsilon>0$ such that, $\left|t-t_{0}\right|<\delta \Longrightarrow D\left(f(t), f\left(t_{0}\right)\right)<\varepsilon$ exists, $f$ is said to be continuous [20, 21].

Definition 2.5 Let $u, v \in \mathbb{E}^{1}$. If there exists $w \in \mathbb{E}^{1}$ such that $u=v+w$, then $w$ is called the H-difference of $u, v$ and it is denoted by $u \ominus v$ [9].

Definition 2.6 Let $a, b \in \mathbb{R}$ and $f:(a, b) \rightarrow \mathbb{E}^{1}$ and $t_{0} \in(a, b)$. We define the $n$-th order differential of $f$ as follows: We say that $f$ is strongly generalized differentiable of $n$-th order at $t_{0}$, if there exists an element $f^{(s)}\left(t_{0}\right) \in \mathbb{E}^{1} \quad \forall s=1, \ldots, n$ such that

(i) for all $h>0$ sufficiently close to 0 , there exist $f^{(s-1)}\left(t_{0}+h\right) \ominus f^{(s-1)}\left(t_{0}\right), f^{(s-1)} \quad\left(t_{0}\right) \ominus$ $f^{(s-1)}\left(t_{0}-h\right)$ and the limits

$$
\begin{aligned}
& \lim _{h \rightarrow 0^{+}} \frac{f^{(s-1)}\left(t_{0}+h\right) \ominus f^{(s-1)}\left(t_{0}\right)}{h} \\
& \quad=\lim _{h \rightarrow 0^{+}} \frac{f^{(s-1)}\left(t_{0}\right) \ominus f^{(s-1)}\left(t_{0}-h\right)}{h}=f^{(s)}\left(t_{0}\right),
\end{aligned}
$$

or

(ii) for all $h>0$ sufficiently close to 0 , there exist $f^{(s-1)}\left(t_{0}-h\right) \ominus f^{(s-1)}\left(t_{0}\right), f^{(s-1)}\left(t_{0}\right) \ominus f^{(s-1)}\left(t_{0}+\right.$ h) and the limits

$$
\begin{aligned}
& \lim _{h \rightarrow 0^{+}} \frac{f^{(s-1)}\left(t_{0}-h\right) \ominus f^{(s-1)}\left(t_{0}\right)}{-h} \\
& \quad=\lim _{h \rightarrow 0^{+}} \frac{f^{(s-1)}\left(t_{0}\right) \ominus f^{(s-1)}\left(t_{0}+h\right)}{-h}=f^{(s)}\left(t_{0}\right),
\end{aligned}
$$

or (iii) for all $h>0$ sufficiently close to 0 , there exist $f^{(s-1)}\left(t_{0}+h\right) \ominus f^{(s-1)}\left(t_{0}\right), \quad f^{(s-1)}\left(t_{0}-h\right) \ominus f^{(s-1)}$

$\left(t_{0}\right)$ and the limits

$$
\begin{aligned}
& \lim _{h \rightarrow 0^{+}} \frac{f^{(s-1)}\left(t_{0}+h\right) \ominus f^{(s-1)}\left(t_{0}\right)}{h} \\
& \quad=\lim _{h \rightarrow 0^{+}} \frac{f^{(s-1)}\left(t_{0}-h\right) \ominus f^{(s-1)}\left(t_{0}\right)}{-h}=f^{(s)}\left(t_{0}\right),
\end{aligned}
$$

or

(iv) for all $h>0$ sufficiently close to 0 , there exist $f^{(s-1)}\left(t_{0}\right) \ominus f^{(s-1)}\left(t_{0}+h\right), f^{(s-1)}\left(t_{0}\right) \ominus f^{(s-1)}\left(t_{0}-\right.$ h) and the limits

$$
\begin{aligned}
& \lim _{h \rightarrow 0^{+}} \frac{f^{(s-1)}\left(t_{0}\right) \ominus f^{(s-1)}\left(t_{0}+h\right)}{-h} \\
& \quad=\lim _{h \rightarrow 0^{+}} \frac{f^{(s-1)}\left(t_{0}\right) \ominus f^{(s-1)}\left(t_{0}-h\right)}{h}=f^{(s)}\left(t_{0}\right),
\end{aligned}
$$

( $h$ and $(-h)$ at denominators mean $\frac{1}{h}$. and $-\frac{1}{h}$. respectively $\forall s=1, \ldots, n)[9,11,12]$.

Theorem 2.7 Let $f:(a, b) \rightarrow \mathbb{E}^{1}$ be strongly generalized differentiable on each point $t \in(a, b)$ in the sense of Definition 2.5, (iii) or (iv). Then $f^{\prime}(x) \in \mathbb{R}$ for all $t \in(a, b)$ (see [9]).

Theorem 2.8 Let $f: \mathbb{R}^{1} \longrightarrow \mathbb{E}^{1}$ be a function and denote $f(t)=(f(t, r), \bar{f}(t, r))$, for each $r \in[0,1]$. Then

(1) If $f$ is differentiable in the first form $(i)$, then $f(t, r)$ and $\bar{f}(t, r)$ are differentiable functions and $f^{\prime}(t)=\left(f^{\prime}(t, r), \overline{f^{\prime}}(t, r)\right)$,

(2) If $f$ is differentiable in the second form (ii), then $f(t, r)$ and $\bar{f}(t, r)$ are differentiable functions and $f^{\prime}(t)=\left(\overline{f^{\prime}}(t, r), \underline{f^{\prime}}(t, r)\right)($ see $[11])$.

Remark 2.9 Note that by the above definition, a fuzzy function is i-differentiable or ii-differentiable of order $n$ if $f^{(s)}$ for $s=1, \ldots, n$ is i-differentiable or ii-differentiable. It is possible that the different orders have different kind $i$ or ii differentiability.

\section{Main idea}

In order to describe the HAM for Eq. (1), we consider the following equation:

$\widetilde{N}[\widetilde{u}(x, t)]=\widetilde{u}_{t t}+\alpha \widetilde{u}_{x x}+\beta\left(\widetilde{u}^{2}\right)_{x x}-\widetilde{u}_{x x x x}=\widetilde{0}$,

According to the parametric form of fuzzy numbers, we consider Eq. (4) in the following form:

$$
N=\left(\begin{array}{l}
\underline{N}[u(x, t)] \\
\bar{N}[u(x, t)]
\end{array}\right)=\left(\begin{array}{l}
0 \\
0
\end{array}\right), \quad u(x, t)=\left(\begin{array}{l}
\underline{u}(x, t) \\
\bar{u}(x, t)
\end{array}\right) .
$$


At first, we construct the zeroth-order deformation system. $(I-Q) L\left[\phi(x, t, r ; Q)-u_{0}(x, t, r)\right]=Q H h N[\phi(x, t, r ; Q)]$,

where $I$ is the identity matrix, $L=\left(\begin{array}{cc}L_{1} & 0 \\ 0 & L_{2}\end{array}\right)$ is an auxiliary linear operator matrix, $H(x, t, r)=$ $\left(\begin{array}{cc}H_{1}(x, t, r) & 0 \\ 0 & H_{2}(x, t, r)\end{array}\right)$ is an auxiliary function matrix, $h=\left(\begin{array}{cc}h_{1} & 0 \\ 0 & h_{2}\end{array}\right)$ is an auxiliary parameter matrix, $\phi(x, t ; Q)=\left(\begin{array}{c}\phi(x, t, r ; q) \\ \bar{\phi}(x, t, r ; q)\end{array}\right)$ is an unknown function matrix, $u_{0}(x, t, r)$ is an initial guess of the vector $u(x, t, r)$ and $Q=\left(\begin{array}{cc}q & 0 \\ 0 & q\end{array}\right), \quad 0 \leq q \leq 1$, is a diagonal matrix which denotes the embedding parameter matrix. It is obvious, when the $q$, increases from 0 to 1 or in other word the embedding parameter matrix changes from $Q=\overline{0}$ to $Q=I$, the solution of system of equations (5) changes from $\phi(x, t, r ; \overline{0})=u_{0}(x, t, r)$ to $\phi(x, t, r ; I)=u(x, t, r)$. Therefore, $\phi(x, t, r)$ varies from the initial guess $u_{0}(x, t, r)$ to the exact solution $u(x, t, r)$ of the system.

We consider $\phi(x, t, r ; Q)$ in the following matrix expansion form,

$\phi(x, t, r ; Q)=u_{0}(x, t, r)+\sum_{m=1}^{+\infty} Q^{m} u_{m}(x, t, r)$

where

$u_{m}(x, t, r)=\frac{1}{m !}\left(\begin{array}{l}\left.\frac{\partial^{m} \underline{\phi}(x, t, r ; q)}{\partial q^{m}}\right|_{q=0} \\ \left.\frac{\partial^{m} \bar{\phi}(x, t, r ; q)}{\partial q^{m}}\right|_{q=0} .\end{array}\right)$.

The convergence of the vector series (6) depends upon the auxiliary parameter matrix $h$, if it is convergent at $Q=I$, we have

$u(x, t, r)=u_{0}(x, t, r)+\sum_{m=1}^{+\infty} u_{m}(x, t, r)$.

Now, we define the vectors,

$\vec{u}_{k}(x, t, r)=\left\{u_{0}(x, t, r), \ldots, u_{k}(x, t, r)\right\}$,

where

$u_{i}=\left(\begin{array}{c}\underline{u}_{i}(x, t, r) \\ \bar{u}_{i}(x, t, r)\end{array}\right), \quad i=0, \ldots, k$.

The $m$-th order deformation system can be written as
$L\left[u_{m}(x, t, r)-\chi_{m} u_{(m-1)}(x, t, r)\right]=h H R_{m}\left(\vec{u}_{m-1}(x, t, r)\right)$,

where

$$
\begin{aligned}
& R_{m}\left(\vec{u}_{m-1}\right)=\frac{1}{(m-1) !}\left(\begin{array}{l}
\left.\frac{\partial^{m-1} \underline{\underline{N}}[\phi(x, t ; Q)]}{\partial q^{m-1}}\right|_{Q=\overline{0}} \\
\left.\frac{\partial^{m-1} \bar{N}[\phi(x, t ; Q)]}{\partial q^{m-1}}\right|_{Q=\overline{0}}
\end{array}\right), \\
& \chi_{m}= \begin{cases}\overline{0}, & m \leq 1, \\
I, & m>1 .\end{cases}
\end{aligned}
$$

If we consider $L=\left(\begin{array}{cc}\frac{\partial^{2}}{\partial t^{2}} & 0 \\ 0 & \frac{\partial^{2}}{\partial t^{2}}\end{array}\right)$, then we have,

$$
\begin{aligned}
u_{m}(t, r)= & \chi_{m} u_{m-1}(t, r)+L^{-1}\left[\operatorname{HhR} R_{m}\left(\vec{u}_{m-1}\right)\right] \\
= & \chi_{m} u_{m-1}(t, r) \\
& +\left(\begin{array}{cc}
h_{1} & 0 \\
0 & h_{2}
\end{array}\right)\left(\begin{array}{cc}
\int_{0}^{\tau} \int_{0}^{t} d \theta d \tau & 0 \\
0 & \int_{0}^{\tau} \int_{0}^{t} d \theta d \tau
\end{array}\right) \\
& \times\left[\left(\begin{array}{cc}
H_{1} & 0 \\
0 & H_{2}
\end{array}\right) R_{m}\left(\vec{u}_{m-1}\right)\right] .
\end{aligned}
$$

Theorem 2.10 If the series solution (8) of problem (1) obtained from the HAM and also the series $\sum_{m=0}^{+\infty} \frac{\partial^{2}}{\partial t^{2}} \underline{u}_{m}$, $\sum_{m=0}^{+\infty} \frac{\partial^{2}}{\partial x^{2}} \underline{u}_{m}, \quad \sum_{m=0}^{+\infty} \frac{\partial^{2}}{\partial x^{2}} \underline{u}_{m}^{2}, \sum_{m=0}^{+\infty} \frac{\partial^{4}}{\partial x^{4}} \underline{u}_{m}, \quad \sum_{m=0}^{+\infty} \frac{\partial^{2}}{\partial t^{2}} \bar{u}_{m}$, $\sum_{m=0}^{+\infty} \frac{\partial^{2}}{\partial x^{2}} \bar{u}_{m}, \sum_{m=0}^{+\infty} \frac{\partial^{2}}{\partial x^{2}} \bar{u}_{m}^{2}$, and $\sum_{m=0}^{+\infty} \frac{\partial^{4}}{\partial x^{4}} \bar{u}_{m}$ are convergent, and also $\underline{0}_{m}$ and $\overline{0}_{m}$ converge to the $\underline{0}$ and $\overline{0}$ respectively, then (8) converges to the exact solution of the problem (1).

Proof Without loss of generality, we suppose $u$ be i-differentiable with respect to the $x, t$ and also it be a positive fuzzy number $(\forall t \in[0, T])$. Therefore, we can write Eq. (1) in the following form:

$$
\begin{aligned}
& \widetilde{u}_{t t}+\left(\alpha^{+}-\alpha^{-}\right) \widetilde{u}_{x x}+\left(\beta^{+}-\beta^{-}\right)\left(\widetilde{u}^{2}\right)_{x x}-\widetilde{u}_{x x x x}=\widetilde{0} \\
& 0 \leq t \leq T, x>0
\end{aligned}
$$

where $\alpha^{+}, \alpha^{-}, \beta^{+}, \beta^{-} \geq 0$.

Therefore, we have

$$
\begin{aligned}
& \left(\begin{array}{l}
\underline{N}[u(x, t, r)] \\
\bar{N}[u(x, t, r)]
\end{array}\right) \\
& =\left(\begin{array}{l}
\underline{u}_{t t}+\alpha^{+} \underline{u}_{x x}-\alpha^{-} \bar{u}_{x x}+\beta^{+} \underline{u}_{x x}^{2}-\beta^{-} \bar{u}_{x x}^{2}-\bar{u}_{x x x x}-\underline{0} \\
\bar{u}_{t t}+\alpha^{+} \bar{u}_{x x}-\alpha^{-} \underline{u}_{x x}+\beta^{+} \bar{u}_{x x}^{2}-\beta^{-} \underline{u}_{x x}^{2}-\underline{u}_{x x x}-\overline{0}
\end{array}\right) \\
& =\left(\begin{array}{l}
0 \\
0
\end{array}\right) \text {. }
\end{aligned}
$$


If the series

$\sum_{m=0}^{+\infty} u_{m}(x, t, r)=\left(\begin{array}{c}\sum_{m=0}^{+\infty} \underline{u}_{m}(x, t, r) \\ \sum_{m=0}^{+\infty} \bar{u}_{m}(x, t, r)\end{array}\right)$

converges, we assume

$u(x, t, r)=\sum_{m=0}^{+\infty} u_{m}(x, t, r)$

whereIn general, the series

$$
\lim _{m \rightarrow+\infty} u_{m}(x, t, r)=\overrightarrow{0}
$$

We write

$\sum_{m=1}^{+\infty}\left[R_{m}\left(\vec{u}_{m-1}\right)\right]=$

$$
\begin{aligned}
& \sum_{m=1}^{+\infty} L\left[u_{m}(x, t, r)-\chi_{m} u_{m-1}(x, t, r)\right] \\
& \quad=L \sum_{m=1}^{+\infty}\left[u_{m}(x, t, r)-\chi_{m} u_{m-1}(x, t, r)\right]=\overrightarrow{0} .
\end{aligned}
$$

From above expression and Eq. (13), we obtain

$$
\sum_{m=1}^{+\infty} L\left[u_{m}(x, t, r)-\chi_{m} u_{m-1}(x, t, r)\right]=h H(x, t) \sum_{m=1}^{+\infty}\left[R_{m}\left(\vec{u}_{m-1}\right)\right] .
$$

Since $h \neq 0$ and $H(x, t) \neq 0$, we have

$\sum_{m=1}^{+\infty}\left[R_{m}\left(\vec{u}_{m-1}\right)\right]=\overrightarrow{0}$

From (12), it holds

$$
\left(\begin{array}{c}
\frac{\partial^{2}}{\partial t^{2}} \sum_{m=1}^{+\infty} \underline{u}_{m-1}+\alpha^{+} \frac{\partial^{2}}{\partial x^{2}} \sum_{m=1}^{+\infty} \underline{u}_{m-1}-\alpha^{-} \frac{\partial^{2}}{\partial x^{2}} \sum_{m=1}^{+\infty} \bar{u}_{m-1}+\beta^{+} \frac{\partial^{2}}{x^{2}}\left[\sum_{m=1}^{+\infty} \sum_{i=0}^{m-1} \underline{u}_{i} \underline{u}_{m-i-1}\right] \\
-\beta^{-} \frac{\partial^{2}}{x^{2}}\left[\sum_{m=1}^{+\infty} \sum_{i=0}^{m-1} \bar{u}_{i} \bar{u}_{m-i-1}\right]-\frac{\partial^{4}}{\partial x^{4}} \sum_{m=1}^{+\infty} \bar{u}_{m-1}-\sum_{m=0}^{+\infty} \underline{0}_{m} \\
\frac{\partial^{2}}{\partial t^{2}} \sum_{m=1}^{+\infty} \bar{u}_{m-1}+\alpha^{+} \frac{\partial^{2}}{\partial x^{2}} \sum_{m=1}^{+\infty} \bar{u}_{m-1}-\alpha^{-} \frac{\partial^{2}}{\partial x^{2}} \sum_{m=1}^{+\infty} \underline{u}_{m-1}+\beta^{+} \frac{\partial^{2}}{x^{2}}\left[\sum_{m=1}^{+\infty} \sum_{i=0}^{m-1} \bar{u}_{i} \bar{u}_{m-i-1}\right] \\
-\beta^{-} \frac{\partial^{2}}{x^{2}}\left[\sum_{m=1}^{+\infty} \sum_{i=0}^{m-1} \underline{u}_{i} \underline{u}_{m-i-1}\right]-\frac{\partial^{4}}{\partial x^{4}} \sum_{m=1}^{+\infty} \underline{u}_{m-1}-\sum_{m=0}^{+\infty} \overline{0}_{m}
\end{array}\right) .
$$

$$
\begin{aligned}
& \sum_{m=1}^{n}\left[u_{m}(x, t, r)-\chi_{m} u_{m-1}(x, t, r)\right] \\
& \quad=u_{1}+\left(u_{2}-u_{1}\right)+\left(u_{3}-u_{2}\right)+\cdots+\left(u_{n}-u_{n-1}\right) \\
& \quad=u_{n}(x, t, r)
\end{aligned}
$$

using (16), we have

$\sum_{m=1}^{+\infty}\left[u_{m}(x, t, r)-\chi_{m} u_{m-1}(x, t, r)\right]=\lim _{n \rightarrow+\infty} u_{n}(x, t, r)=\overrightarrow{0}$

According to the definition of the operator $L$, we can write
We consider $\sum_{m=1}^{+\infty} \sum_{i=0}^{m-1} \underline{u}_{i} \underline{u}_{m-i-1}$, we have

$$
\begin{aligned}
\sum_{m=1}^{+\infty} \sum_{i=0}^{m-1} \underline{u}_{i} \underline{u}_{m-i-1}= & \sum_{i=0}^{+\infty} \sum_{m=i+1}^{+\infty} \underline{u}_{i} \underline{u}_{m-i-1}=\sum_{i=0}^{+\infty} \underline{u}_{i} \sum_{m=i+1}^{+\infty} \underline{u}_{m-i-1} \\
& =\sum_{i=0}^{+\infty} \underline{u}_{i} \sum_{m=0}^{+\infty} \underline{u}_{m} .
\end{aligned}
$$

Similarly for next elements. Finally, $\sum_{m=1}^{+\infty}\left[R_{m}\left(\vec{u}_{m-1}\right)\right]$ 


$$
\begin{aligned}
& =\left(\begin{array}{c}
\frac{\partial^{2}}{\partial t^{2}} \sum_{m=0}^{+\infty} \underline{u}_{m}+\alpha^{+} \frac{\partial^{2}}{\partial x^{2}} \sum_{m=0}^{+\infty} \underline{u}_{m}-\alpha^{-} \frac{\partial^{2}}{\partial x^{2}} \sum_{m=0}^{+\infty} \bar{u}_{m}+\beta^{+} \frac{\partial^{2}}{x^{2}}\left[\sum_{i=0}^{+\infty} \underline{u}_{i} \sum_{m=0}^{+\infty} \underline{u}_{m}\right] \\
-\beta^{-} \frac{\partial^{2}}{x^{2}}\left[\sum_{i=0}^{+\infty} \bar{u}_{i} \sum_{m=0}^{+\infty} \bar{u}_{m}\right]-\frac{\partial^{4}}{\partial x^{4}} \sum_{m=0}^{+\infty} \bar{u}_{m}-\sum_{m=0}^{+\infty} \underline{0}_{m} \\
\frac{\partial^{2}}{\partial t^{2}} \sum_{m=0}^{+\infty} \bar{u}_{m}+\alpha^{+} \frac{\partial^{2}}{\partial x^{2}} \sum_{m=0}^{+\infty} \bar{u}_{m}-\alpha^{-} \frac{\partial^{2}}{\partial x^{2}} \sum_{m=0}^{+\infty} \underline{u}_{m}+\beta^{+} \frac{\partial^{2}}{x^{2}}\left[\sum_{i=0}^{+\infty} \bar{u}_{i} \sum_{m=0}^{+\infty} \bar{u}_{m}\right] \\
-\beta^{-} \frac{\partial^{2}}{x^{2}}\left[\sum_{i=0}^{+\infty} \underline{u}_{i} \sum_{m=0}^{+\infty} \underline{u}_{m}\right]-\frac{\partial^{4}}{\partial x^{4}} \sum_{m=0}^{+\infty} \underline{u}_{m}-\sum_{m=0}^{+\infty} \overline{0}_{m}
\end{array}\right) \\
& =\overrightarrow{0} .
\end{aligned}
$$

Therefore,

$$
\begin{aligned}
& \left(\begin{array}{l}
\underline{u}_{t t}+\alpha^{+} \underline{u}_{x x}-\alpha^{-} \bar{u}_{x x}+\beta^{+} \underline{u}_{x x}^{2}-\beta^{-} \bar{u}_{x x}^{2}-\bar{u}_{x x x x}-\underline{0} \\
\bar{u}_{t t}+\alpha^{+} \bar{u}_{x x}-\alpha^{-} \underline{u}_{x x}+\beta^{+} \bar{u}_{x x}^{2}-\beta^{-} \underline{u}_{x x}^{2}-\underline{u}_{x x x x}-\overline{0}
\end{array}\right) \\
& \quad=\left(\begin{array}{l}
0 \\
0
\end{array}\right),
\end{aligned}
$$

and it means that

$\widetilde{u}_{t t}+\alpha \widetilde{u}_{x x}+\beta\left(\widetilde{u}^{2}\right)_{x x}-\widetilde{u}_{x x x x}=\widetilde{0}$.

\section{Test examples}

In this section, we solve two sample examples to illustrate the applicability of the proposed method. The results are provided by Maple.

Example 3.1 We consider the following fuzzy Boussinesq equation

$\widetilde{u}_{t t}-\widetilde{u}_{x x}+\left(\widetilde{u}^{2}\right)_{x x}-\widetilde{u}_{x x x x}=\widetilde{0}$,

with the initial conditions:

$\widetilde{u}(x, 0)=(r, 3-2 r) \frac{6}{x^{2}}, \widetilde{u}_{t}(x, 0)=(r, 3-2 r) \frac{-12}{x^{3}}$, where $\quad \tilde{0}=(3 r-3,3-3 r) \widehat{u}_{x x}+\left(r^{2}-(3-2 r),(3-\right.$ $\left.2 r)^{2}-(r)\right) \widehat{u}_{x x x x}$ and $\widehat{u}$ is the solution of crisp case of the equation.

We suppose, $\widetilde{u}_{t}$ be i-differentiable with respect to the $t$ and $\widetilde{u}_{x}, \widetilde{u}_{x}^{2}$ and $\widetilde{u}_{x x x}$ are i-differentiable with respect to the $x$. Also $\widetilde{u}$ be a positive fuzzy number $(\forall t \in[0, T], x>0)$, therefore we have

$$
\begin{aligned}
& \left(\begin{array}{l}
\underline{u}_{t t}-\bar{u}_{x x}+\left(\underline{u}^{2}\right)_{x x}-\bar{u}_{x x x x}-(3 r-3) \widehat{u}_{x x}-\left(r^{2}-(3-2 r)\right) \widehat{u}_{x x x x} \\
\bar{u}_{t t}-\underline{u}_{x x}+\left(\bar{u}^{2}\right)_{x x}-\underline{u}_{x x x x}-(3-3 r) \widehat{u}_{x x}-\left((3-2 r)^{2}-r\right) \widehat{u}_{x x x x}
\end{array}\right) \\
& \quad=\left(\begin{array}{l}
0 \\
0
\end{array}\right) .
\end{aligned}
$$

We consider $H=I$ and $h=-I$, and also, we choose the initial approximate as $\left(\begin{array}{c}r \frac{6 x-12 t}{x^{3}} \\ (3-2 r) \frac{6 x-12 t}{x^{3}}\end{array}\right)$. According to Eq. (13), we have

$$
\left.R_{m}\left(\vec{u}_{m-1}\right)\right]=\left(\begin{array}{c}
\frac{\partial^{2}}{\partial t^{2}} \underline{u}_{m-1}-\frac{\partial^{2}}{\partial x^{2}} \sum_{m=1}^{+\infty} \bar{u}_{m-1}+\frac{\partial^{2}}{x^{2}}\left[\sum_{i=0}^{m-1} \underline{u}_{i} \underline{u}_{m-i-1}\right]-\frac{\partial^{4}}{\partial x^{u^{\prime}}} \bar{u}_{m-1} \\
-(3 r-3) \frac{\partial^{2}}{x^{2}} \widehat{u}-\left(r^{2}-(3-2 r)\right) \frac{\partial^{4}}{\partial x^{4}} \widehat{u} \\
\frac{\partial^{2}}{\partial t^{2}} \bar{u}_{m-1}-\frac{\partial^{2}}{\partial x^{2}} \sum_{m=1}^{+\infty} \underline{u}_{m-1}+\frac{\partial^{2}}{x^{2}}\left[\sum_{i=0}^{m-1} \bar{u}_{i} \bar{u}_{m-i-1}\right]-\frac{\partial^{4}}{\partial x^{4}} \underline{u}_{m-1} \\
-(3-3 r) \frac{\partial^{2}}{x^{2}} \widehat{u}-\left((3-2 r)^{2}-r\right) \frac{\partial^{4}}{\partial x^{4}} \widehat{u}
\end{array}\right) .
$$

Therefore,

$$
u_{0}=\left(\begin{array}{c}
r\left(\frac{6}{x^{2}}-\frac{12 t}{x^{3}}\right) \\
(3-2 r)\left(\frac{6}{x^{2}}-\frac{12 t}{x^{3}}\right)
\end{array}\right)
$$

$$
\begin{gathered}
u_{1}=\left(\begin{array}{c}
-\frac{18 r t^{2}}{x^{4}}-\frac{24 r t^{3}}{x^{5}}-\frac{504 r^{2} t^{4}}{x^{8}} \\
-\frac{(72 r-108) t^{2}}{2 x^{4}}-\frac{(-144 r+216) t^{3}}{3 x^{5}}-\frac{3\left(1512+672 r^{2}-2016 r\right) t^{4}}{x^{8}}
\end{array}\right) \\
u_{2}=\left(\begin{array}{c}
-\frac{30 r t^{4}}{x^{6}}-\frac{24 r t^{5}}{x^{7}}+\frac{504 r^{2} t^{4}}{x^{8}}+\cdots \\
-\frac{(240 r-360) t^{4}}{4 x^{6}}-\frac{(540-360 r) t^{5}}{5 x^{7}}+\frac{3\left(1512+672 r^{2}-2016 r\right) t^{4}}{x^{8}}+\cdots
\end{array}\right)
\end{gathered}
$$


In general, the series solution is given by

$$
\begin{aligned}
u(x, t) & =\sum_{n=0}^{\infty} u_{n}(x, t) \\
& =\left(\begin{array}{c}
r\left(\frac{6}{x^{2}}-\frac{12}{x^{3}} t+\frac{18}{x^{4}} t^{2}-\frac{24}{x^{5}} t^{3}+\frac{30}{x^{6}} t^{4}+\cdots\right) \\
(3-2 r)\left(\frac{6}{x^{2}}-\frac{12}{x^{3}} t+\frac{18}{x^{4}} t^{2}-\frac{24}{x^{5}} t^{3}+\frac{30}{x^{6}} t^{4}+\cdots\right)
\end{array}\right) .
\end{aligned}
$$

We suppose $\widetilde{u}_{t}$ be ii-differentiable with respect to the $t, \widetilde{u}_{x}, \widetilde{u}_{x x x}$ are ii-differentiable with respect to the $x$ and $\widetilde{u}_{x}^{2}$ is i-differentiable with respect to the $x$. Also $\widetilde{u}$ be a negative fuzzy number $(\forall t \in[0, T], x>0)$, therefore we have

$$
\left(\begin{array}{c}
\bar{u}_{t t}-\underline{u}_{x x}-\left(\bar{u}^{2}\right)_{x x}-\underline{u}_{x x x x}-\left(\frac{3 r}{2}-\frac{3}{2}\right)\left(-\widehat{u}_{x x}\right)-\left(\left(\frac{r}{2}+\frac{1}{2}\right)-(2-r)^{2}\right)\left(-\widehat{u}_{x x x x}\right) \\
\underline{u}_{t t}-\bar{u}_{x x}-\left(\underline{u}^{2}\right)_{x x}-\bar{u}_{x x x x}-\left(\frac{-3 r}{2}+\frac{3}{2}\right)\left(-\widehat{u}_{x x}\right)-\left((2-r)-\left(\frac{r}{2}+\frac{1}{2}\right)^{2}\right)\left(-\widehat{u}_{x x x x}\right)
\end{array}\right)=\left(\begin{array}{l}
0 \\
0
\end{array}\right) .
$$

That gives the exact solution

$$
\left(\begin{array}{c}
\frac{6 r}{(x+t)^{2}} \\
\frac{6(3-2 r)}{(x+t)^{2}}
\end{array}\right)
$$

Therefore, $\widetilde{u}=(r, 3-2 r) \frac{6}{(x+t)^{2}}$ is the exact solution of the fuzzy differential equation.

Example 3.2 We consider the following fuzzy Boussing equation

$$
\widetilde{u}_{t t}-\widetilde{u}_{x x}-\left(\widetilde{u}^{2}\right)_{x x}-\widetilde{u}_{x x x x}=\widetilde{0},
$$

with the initial conditions:

$$
\begin{aligned}
\widetilde{u}(x, 0) & =\left(\frac{r}{2}+\frac{1}{2}, 2-r\right) \frac{-6}{x^{2}} \\
\widetilde{u}_{t}(x, 0) & =\left(\frac{r}{2}+\frac{1}{2}, 2-r\right) \frac{12}{x^{3}}
\end{aligned}
$$

where $\quad \widetilde{0}=\left(\frac{3 r}{2}-\frac{3}{2},-\frac{3 r}{2}+\frac{3}{2}\right)\left(-\widehat{u}_{x x}\right)+\left(\left(\frac{r}{2}+\frac{1}{2}\right)-(2-\right.$ $\left.r)^{2},(2-r)-\left(\frac{r}{2}+\frac{1}{2}\right)^{2}\right)\left(-\widehat{u}_{x x x x}\right)$ and $\widehat{u}$ is the solution of crisp case of the equation.
We consider $H=I$ and $h=-I$, and also, we choose the initial approximate as $\left(\begin{array}{l}\left(\frac{r}{2}+\frac{1}{2}\right) \frac{-6 x+12 t}{x^{3}} \\ (2-r) \frac{-6 x+12 t}{x^{3}}\end{array}\right)$. According to Eq. (13), we have

$$
\begin{aligned}
& \left.R_{m}\left(\vec{u}_{m-1}\right)\right] \\
& =\left(\begin{array}{c}
\frac{\partial^{2}}{\partial t^{2}} \underline{u}_{m-1}-\frac{\partial^{2}}{\partial x^{2}} \sum_{m=1}^{+\infty} \bar{u}_{m-1}-\frac{\partial^{2}}{x^{2}}\left[\sum_{i=0}^{m-1} \bar{v}_{i} \bar{v}_{m-i-1}\right]-\frac{\partial^{4}}{\partial x^{4}} \underline{u}_{m-1} \\
-\left(\frac{3}{2} r-\frac{3}{2}\right) \frac{\partial^{2}}{x^{2}}(-\widehat{u})-\left(\left(\frac{r}{2}+\frac{1}{2}\right)-(2-r)^{2}\right) \frac{\partial^{4}}{\partial x^{4}}(-\widehat{u}) \\
\frac{\partial^{2}}{\partial t^{2}} \underline{u}_{m-1}-\frac{\partial^{2}}{\partial x^{2}} \sum_{m=1}^{+\infty} \bar{u}_{m-1}-\frac{\partial^{2}}{x^{2}}\left[\sum_{i=0}^{m-1} \underline{u}_{i} \underline{u}_{m-i-1}\right]+\frac{\partial^{4}}{\partial x^{4}} \bar{u}_{m-1} \\
-\left(\frac{3}{2}-\frac{3}{2} r\right) \frac{\partial^{2}}{x^{2}}(-\widehat{u})-\left((2-r)-\left(\frac{r}{2}+\frac{1}{2}\right)^{2}\right) \frac{\partial^{4}}{\partial x^{4}}(-\widehat{u})
\end{array}\right) .
\end{aligned}
$$

Therefore,

$$
u_{0}=\left(\begin{array}{c}
\left(\frac{r}{2}+\frac{1}{2}\right)\left(\frac{6}{x^{2}}-\frac{12 t}{x^{3}}\right) \\
(2-r)\left(\frac{6}{x^{2}}-\frac{12 t}{x^{3}}\right)
\end{array}\right)
$$




$$
\begin{gathered}
u_{1}=\left(\begin{array}{c}
-\frac{(18 r+18) t^{2}}{2 x^{4}}-\frac{(36-36 r) t^{3}}{3 x^{5}}+\frac{3\left(42+48 r+42 r^{2}\right) t^{4}}{x^{8}} \\
-\frac{(-36 r+72) t^{2}}{2 x^{4}}-\frac{(144 r+72) t^{3}}{3 x^{5}}+\frac{3\left(1344-1344 r+336 r^{2}\right) t^{4}}{2 x^{8}}
\end{array}\right) \\
u_{2}=\left(\begin{array}{c}
-\frac{(60 r+60) t^{4}}{4 x^{6}}-\frac{(90 r-90) t^{5}}{5 x^{7}}-\frac{3\left(42+48 r+42 r^{2}\right) t^{4}}{x^{8}}+\cdots \\
-\frac{(240-120 r) t^{4}}{4 x^{6}}-\frac{(180 r-360) t^{5}}{5 x^{7}}-\frac{3\left(1344-1344 r+336 r^{2}\right) t^{4}}{2 x^{8}}+\cdots
\end{array}\right) \\
\vdots
\end{gathered}
$$

In general, the series solution is given by

$$
\begin{aligned}
u(x, t) & =\sum_{n=0}^{\infty} u_{n}(x, t) \\
= & \left(\begin{array}{c}
\left(\frac{r}{2}+\frac{1}{2}\right)\left(-\frac{6}{x^{2}}+\frac{12}{x^{3}} t-\frac{18}{x^{4}} t^{2}+\frac{24}{x^{5}} t^{3}-\frac{30}{x^{6}} t^{4}+\cdots\right) \\
(2-r)\left(-\frac{6}{x^{2}}+\frac{12}{x^{3}} t-\frac{18}{x^{4}} t^{2}+\frac{24}{x^{5}} t^{3}-\frac{30}{x^{6}} t^{4}+\cdots\right)
\end{array}\right) .
\end{aligned}
$$

That gives the exact solution

$$
\left(\begin{array}{c}
\left(\frac{r}{2}+\frac{1}{2}\right) \frac{-6}{(x+t)^{2}} \\
(2-r) \frac{-6}{(x+t)^{2}}
\end{array}\right) .
$$

Therefore, $\widetilde{u}=\left(\frac{r}{2}+\frac{1}{2}, 2-r\right)\left(\frac{-6}{(x+t)^{2}}\right)$ is the exact solution of the fuzzy differential equation.

\section{Conclusion}

In this work, we applied the fuzzy HAM in order to solve the fuzzy Boussinesq equation. For this aim, we considered the parametric form of a fuzzy number and established the deformation equations for two crisp Bousinnesq equations obtained from the proposed method. Also, we presented a theorem to warrant the convergence of the proposed method too. Similar to the discussion in this work, the HAM can be used in order to solve other kinds of fuzzy differential equations as an efficient and proper method.

Acknowledgments The authors would like to thank the anonymous reviewers for their careful reading and suggestions to improve the quality of this work and also are thankful to the Islamic Azad University, Central Tehran branch for their support during this research.
Open Access This article is distributed under the terms of the Creative Commons Attribution 4.0 International License (http:// creativecommons.org/licenses/by/4.0/), which permits unrestricted use, distribution, and reproduction in any medium, provided you give appropriate credit to the original author(s) and the source, provide a link to the Creative Commons license, and indicate if changes were made.

\section{References}

1. Abbasbandy, S., Allahviranloo, T.: Numerical solution of fuzzy differential equation by Taylor method. J. Comput. Methods Appl. Math. 2, 113-124 (2002)

2. Abbasbandy, S., Allahviranloo, T., Loez-Pouso, O., Nieto, J.J.: Numerical methods for fuzzy differential inclusions. J. Comput. Math. Appl. 48, 1633-1641 (2004)

3. Allahviranloo, T., Ahmadi, N., Ahmadi, E.: Numerical solution of fuzzy differential equations by predictor-corrector method. Inf. Sci. 177, 1633-1647 (2007)

4. Allahviranloo, T., Ahmadi, E., Ahmadi, A.: $N$-th fuzzy differential equations. Inf. Sci. 178, 1309-1324 (2008)

5. Allahviranloo, T., Kiani, N.A., Motamedi, N.: Solving fuzzy differential equations by differential transformation method. Inf. Sci. 179(7), 956-966 (2009)

6. Allahviranloo, T., Chehlabi, M.: Solving fuzzy differential equations based on the length function properties. Soft Comput. 19, 307-320 (2015)

7. Allahviranloo, T., Salahshour, S.: Euler method for solving hybrid fuzzy differential equation. Soft Comput. 15, 1247-1253 (2011)

8. Buckley, J.J., Feuring, T.: Fuzzy differential equations. Fuzzy Sets Syst. 110, 43-54 (2000)

9. Bede, B., Gal, S.G.: Generalizations of the differentiability of fuzzy number value functions with applications to fuzzy differential equations. Fuzzy Sets Syst. 151, 581-599 (2005)

10. Chalco-Cano, Y., Roman-Flores, H.: Comparision between some approaches to solve the fuzzy differential equations. Fuzzy Sets Syst. 160, 1517-1562 (2009)

11. Chalco-Cano, Y., Roman-Flores, H.: On new solutions of fuzzy differential equations. Chaos Solitons Fractals 38, 112-119 (2006) 
12. Cong-xin, W., Ming, M.: Embedding problem of fuzzy number space: I. Fuzzy Sets Syst. 44, 33-38 (1991)

13. Araghi, M.A.F., Fallahzadeh, A.: On the convergence of the homotopy analysis method for solving the Schrodinger equation. J. Basic Appl. Sci. Res. 2(6), 6076-6083 (2012)

14. Araghi, M.A.F., Fallahzadeh, A.: Explicit series solution of Boussinesq equation by homotopy analysis method. J. Am. Sci. 8(11), 448-452 (2012)

15. Goetschel, R., Voxman, W.: Elementary fuzzy calculus. Fuzzy Sets Syst. 18, 31-43 (1986)

16. Liao, S.J.: Beyond Pertubation: Introduction to the Homotopy Analysis Method. Chapman and Hall/CRC Press, Boca Raton (2003)

17. Liao, S.J.: Notes on the homotopy analysis method: some definitions and theorems. Commun. Nonlinear Sci. Numer. Simul. 14, 983-997 (2009)
18. Lupulescu, V.: On a class of fuzzy functional differential equations. Fuzzy Sets Syst. 160, 1547-1562 (2009)

19. Palligkinis, SCh., Papageorgiou, G., Famelis, ITh: Runge-Kutta methods for fuzzy differential equations. Appl. Math. Comput. 209, 97-105 (2009)

20. Puri, M.L., Ralescu, D.: Fuzzy random variables. J. Math. Anal. Appl. 114, 409-422 (1986)

21. Seikkala, S.: On the fuzzy initial value problem. Fuzzy Set Syst. 24, 319-330 (1987)

22. Zimmerman, H.J.: Fuzzy Set Theory and Its Applications. Kluwer Academic, New York (1996) 